\title{
Ecological and Agronomical Value of Festuca rupicola - Bothriochloa ischaemum Grasslands
}

\author{
Florin PĂCURAR, Ioan ROTAR, Roxana VIDICAN, Anca PLEȘA, Ioana VAIDA*, Anamaria MĂLINAȘ, Vlad \\ STOIAN
}

${ }^{1}$ Faculty of Agriculture, Department of Plant Crops. University of Agricultural Sciences and Veterinary Medicine Cluj-Napoca, Manăștur street, 3-5, 400372,Romania

*corresponding author: ioanavaida@yahoo.com

Bulletin UASVM series Agriculture 73(2)/2016

Print ISSN 1843-5246; Electronic ISSN 1843-5386

DOI 10.15835/buasvmcn-agr: 12416

\begin{abstract}
The purpose of this research is to assess the state of the biodiversity and pastoral value for Festuca rupicolaBothriochloa ischaemum grasslands from the hill area. Also, there has been analyzed the floristic composition and a series of ecological indexes, respectively humidity, soil reaction, temperature and nitrogen. Other aspects taken in account were the agronomical and anthropogenic specters. The analyzed grasslands are placed in the perimeter of the localities: Gilău, Aiton. Jucu and Frata, all from Cluj County.
\end{abstract}

Keywords: biodiversity, Cluj County, Festuca rupicola-Bothriochloa ischaemum, hill area.

\section{INTRODUCTION}

Traditional upland livestock grazing is declining worldwide, leading to concerns about possible impacts on biodiversity (Pollock et al. 2013). Upland livestock farming is an integral part of the culture and history of many pastoral areas worldwide.

The diverse rangelands created by these farming systems are an important complex resource for the rural economy (Gordon et al.2004). The problem they will face is the growing number of small animals that grazed the grasslands, which will become slowly abandoned.

Reductions in overall grazing pressure are also likely to lead to a buildup of rank swards with an increased likelihood of resulting wildfires (Pollock et al. 2013). If there is an overall reduction in grazing pressure, grassland is expected to decline and heathland to increase in abundance, with eventual increases expected in woodland and scrub (Miles1988) or mire vegetation (Fenton 2008).

In Romania important plain and hill grassland areas are grazed. In some areas overgrazing is installed whilein otherareas there is a undergrazing phenomenon. In Transylvania Plain undergrazing is usually present (Păcurar et al., 2015; Rotar et al., 2016) especially in areas with different degrees of tilt and with fried vegetation. One example of grassland in this regard used for grazing is Festuca rupicola - Bothriochloa ischaemum. The purpose of this research is to assess the state of the biodiversity and pastoral value for Festuca rupicola - Bothriochloa ischaemum grasslands fitocoenosis.

\section{MATERIALS AND METHODS}

Study site

Our studies were conducted in 4 different area from The Transylvanian Plain, Romania. The study was performed in 2015 in Gilău, Aiton, Jucu and Frata commune, Cluj County. The area shows a typical plain until hillside climate, the landscape is undulating, with altitudes between 250 and 750 $\mathrm{m}$ above sea level. It is characterized by a high variation of land use and topoclimatic conditions in the area and fine-grained mosaic of different land uses, including substantial amounts of semi 
natural vegetation. The vegetation observations were made on 22 plots.

Data analysis

The floristic composition was interpreted using an improved Braun-Blanquet scale with subdivisions (Păcurar and Rotar, 2014). Sward fodder value was calculated based on species quality score on a scale from 1 (poor) to 9 (excellent), after Dierschke and Briemle (2002), as modified by Păcurar and Rotar (2014). Sward fodder value was performed on a scale from 1 (poor sward, quality dominated by toxic species) to 9 (excellent) after Păcurar and Rotar (2014).

Data regarding the share of economic groups (Poaceae, Cyperaceae-Juncaceae, Fabaceae and other botanical families- AFB), species number were processed by analysis of variance. Plant resistance against interference mechanical, such as mowing, grazing and crushed materialized by value indicator (from 1-9) after Dierschke and Briemle (2002), and the names of appropriate species depending on the category disturbance were taken after Păcurar and Rotar (2014).

Based on data from spectrum it can be calculate the average indicator of a phytocenosis. This may be unweighted or weighted. Assigning a phytocenosis feed is achieved at the expense calculated weighted average indicator value.

Using descriptive statistics (Cristea et al. 2004) analyzes were performed which are divided into two categories: central tendency parameters and indicators of scattering data. In the central tendency parameters included those processes provide a representative value (central) measured for the data stream. There are three estimators that can be used for this purpose: the mean, median and module (http://statisticasociala.tripod.com/ parametri.htm).

\section{RESULTS AND DISCUSSION}

In this descriptive plot has been identified the Festuca rupicola-Bothriochloa ischaemum grassland type, which is part of Festuca rupicola series, feature areas habitats meso-xerofile, thermophilic, with inclined slopes exposed to the south and southwest (T,ucra et al., 1987).

In our case, the type Festuca rupicola -Bothriochloa ischaemum was described in nemoral area, at altitudes between 350 and 750 , generally starting on exhibitions east until the south $\left(90^{\circ}\right.$ $180^{\circ}$ ), land with an average slope of around $26.47^{\circ}$, sometimes reaching up to a maximum inclination of $40^{\circ}$ (Tab. 1). There have been studied 22 grassland plots in the Transylvanian Plain.

Festuca rupicola-Bothriochloa ischaemum type is mostly used by grazing. In this type, woody vegetation cover has an average of $12 \%$ (Tab. 1), the amplitude data is high ( $\max =22.25 \%$ and minimum $=5 \%$ ), and the most common cover being $2 \%$ (Module $=12.5 \%$ ). The stub presence in most cases is lacking entirely.

The situation is similar in the case of rocks and stones. Fallow molehills have a presence, generally about $1.80 \%$. In this grassland type are anthills, but with a small participation 3\% (Tab. 1).

Overall, the herbaceous vegetation coverage is averaging $68.41 \%$ and data distribution is one relatively normal.

The floristic nucleus of this type of grassland is given for edifying species and frequent species. Thus, edifying species $(\mathrm{K}=\mathrm{V})$, for Festuca rupicola -Bothriochloa ischaemum grassland type are: Achillea millefolium (1.81\%), Eryngium campestre (1.72\%), Euphorbia cyparisias (2.32\%), Fragaria vesca (7.53\%) and Plantago media (3.0\%; Tab. 1). The frequent (common) species (K = IV) are just three: Trifolium repens (1.25\%), Plantago lanceolata $(1.83 \%)$ and Salvia pratensis $(2.64 \%$, Tab. 1).

Potential indicator species (K=III) for this studied type of grassland are: Agrimonia eupatoria (1.33\%), Carduus acahthoides (0.70\%), Hieracium pilosella (3.09\%) and Thymus glabrescens (3.68\%).

In this phytocoenosis are 14 accompanying species $(\mathrm{K}=\mathrm{II})$, and 35 random species $(\mathrm{K}=\mathrm{I})$. The Phytodiveristy of this grassland type is given by a 64 species (Tab. 1 ).

Poaceae family makes its presence felt in the sward with an average participation of $54.31 \%$ (Tab. 1), the data having high amplitude (minimum $=2.18 \%$ and maximum $=7.34 \%$; Annex 1 ).

From Poaceae family, besides of the dominant species (Festuca rupicola -Bothriochloa ischaemum) with a big mean abundance (5.71\%) is present Stipa capillata followed by Brachipodium pinnatum (3.5\% coverage), then the species Elymus elongatus $3.10 \%$ (Tab. 1). Regarding the distribution of data only the last two species are normal and symmetric (obliquity $=0.61$ and -0.25), other species of Poaceae having a smaller presence (Annex 1).

Regarding the participation of Cyperaceae and 
Tab. 1. Floristic composition of the type of grassland Festuca rupicola and specific requirement on ecological, agronomic and anthropogenic (B - BioForm, T - temperature, U - humidity, R - soil reaction, $\mathrm{N}$ - nutrition, $\mathrm{C}$ - tolerance of mowing, $\mathrm{P}$ - tolerance of grazing, $\mathrm{S}$ - tolerance of crushed, VF- fodder value, $\mathrm{H}$ - hemerobie, UR - urbanophile, SO - sozological category, $\overline{\mathbf{x}}$-Average, ADm- mean abundance - dominance, K- Constancy)

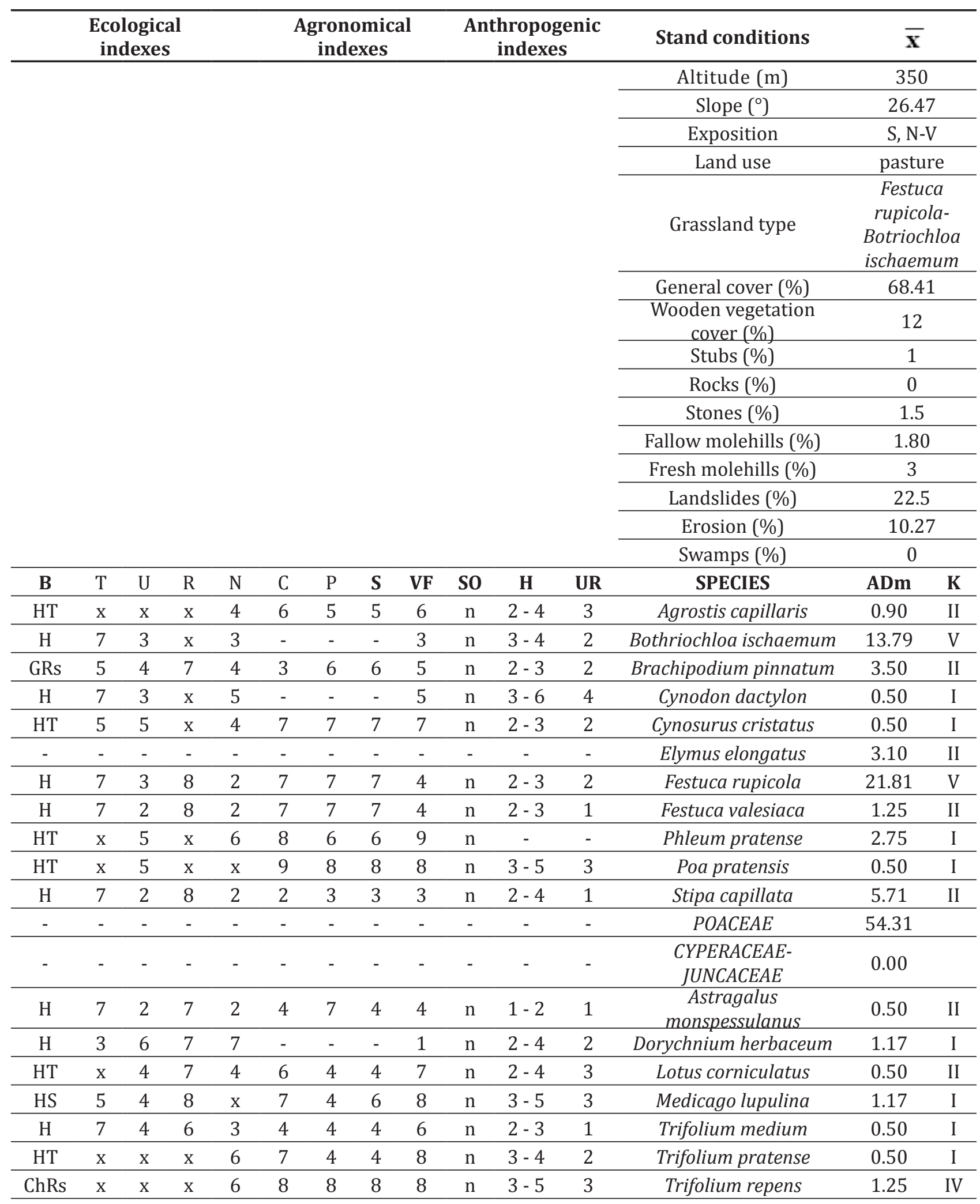




\begin{tabular}{|c|c|c|c|c|c|c|c|c|c|c|c|c|c|c|}
\hline \multicolumn{5}{|c|}{$\begin{array}{l}\text { Ecological } \\
\text { indexes }\end{array}$} & \multicolumn{4}{|c|}{$\begin{array}{c}\text { Agronomical } \\
\text { indexes }\end{array}$} & \multicolumn{3}{|c|}{$\begin{array}{l}\text { Anthropogenic } \\
\text { indexes }\end{array}$} & \multirow{2}{*}{$\begin{array}{c}\text { Stand conditions } \\
\text { FABACEAE }\end{array}$} & \multicolumn{2}{|l|}{$\overline{\mathbf{x}}$} \\
\hline- & - & - & - & - & - & - & - & - & - & - & - & & 5.58 & \\
\hline ChRs & $\mathrm{x}$ & 4 & $\mathrm{x}$ & 5 & 7 & 4 & 5 & 6 & $\mathrm{n}$ & $2-4$ & 3 & Achillea millefolium & 1.81 & $\mathrm{~V}$ \\
\hline HT & 6 & 3 & 7 & 1 & 5 & 8 & 8 & 1 & $\mathrm{n}$ & $2-3$ & 1 & Adonis vernalis & 5.25 & $\mathrm{I}$ \\
\hline HRs & 6 & 4 & 8 & 4 & 3 & 4 & 3 & 3 & $\mathrm{n}$ & $2-3$ & 2 & Agrimonia eupatoria & 1.33 & III \\
\hline HRs & $\mathrm{x}$ & $\mathrm{x}$ & $\mathrm{x}$ & 2 & 4 & 4 & 4 & 4 & $\mathrm{n}$ & $2-3$ & 2 & Campanula serpilifolia & 0.50 & $\mathrm{I}$ \\
\hline TT & 5 & 3 & $\mathrm{x}$ & 8 & 3 & 7 & 3 & 2 & $\mathrm{n}$ & $3-5$ & 3 & Carduus acanthoides & 0.70 & III \\
\hline HRs & $\mathrm{x}$ & $\mathrm{x}$ & $\mathrm{x}$ & $\mathrm{x}$ & 5 & 4 & 4 & 4 & $\mathrm{n}$ & $3-4$ & 2 & Centaurea jacea & 1.50 & I \\
\hline HRs & 6 & 4 & 6 & 6 & 4 & 2 & 2 & 4 & $\mathrm{n}$ & $2-4$ & 1 & Centaurea nigrescens & 0.50 & I \\
\hline $\mathrm{H}$ & $\mathrm{x}$ & 4 & 7 & 3 & - & - & - & 4 & $\mathrm{n}$ & $2-3$ & 1 & Clinopodium vulgare & 0.50 & I \\
\hline GRs & 5 & 6 & 7 & $\mathrm{x}$ & 5 & 9 & 3 & 1 & $\mathrm{n}$ & $2-3$ & 1 & Colchium autumnale & 0.50 & I \\
\hline HRs & 6 & 4 & 8 & 5 & 4 & 5 & 5 & 5 & $\mathrm{n}$ & $3-5$ & 3 & Cichorium intybus & 0.79 & II \\
\hline HRs & 5 & 5 & 6 & 5 & 6 & 2 & 2 & 4 & $\mathrm{n}$ & $3-4$ & 3 & Crepis biennis & 0.50 & I \\
\hline HRs & 6 & 4 & $\mathrm{x}$ & 4 & 6 & 3 & 4 & 5 & $\mathrm{n}$ & $3-5$ & 3 & Daucus carota & 0.50 & $\mathrm{I}$ \\
\hline $\mathrm{H}$ & 6 & 6 & 8 & 7 & 5 & 7 & 3 & 2 & $\mathrm{n}$ & $4-6$ & 3 & Dipsacus fullonum & 1.50 & I \\
\hline- & - & - & - & - & - & - & - & - & - & - & - & Erodium hoefftianum & 0.00 & I \\
\hline $\mathrm{H}$ & 7 & 3 & 8 & 4 & 2 & 4 & 3 & 2 & $\mathrm{n}$ & $2-4$ & 2 & Eryngium campestre & 1.72 & $\mathrm{~V}$ \\
\hline $\mathrm{H}$ & $\mathrm{x}$ & 4 & 8 & $\mathrm{x}$ & 4 & 8 & 7 & 1 & $\mathrm{n}$ & $2-4$ & 2 & Euphorbia cyparissias & 2.32 & $\mathrm{~V}$ \\
\hline HRs & 7 & 4 & $\mathrm{x}$ & 3 & 4 & 2 & 2 & 5 & $\mathrm{n}$ & $2-3$ & 1 & Filipendula hexapetala & 1.50 & II \\
\hline $\mathrm{H}$ & $\mathrm{x}$ & 5 & $\mathrm{x}$ & 6 & 3 & 4 & 4 & 4 & $\mathrm{n}$ & $2-3$ & 2 & Fragaria vesca & 7.53 & $\mathrm{~V}$ \\
\hline HT & 5 & 4 & 7 & 3 & 5 & 4 & 4 & 5 & $\mathrm{n}$ & $2-3$ & 2 & Galium verum & 0.50 & I \\
\hline $\mathrm{HS}$ & $\mathrm{x}$ & 4 & $\mathrm{x}$ & 2 & 4 & 7 & 7 & 4 & $\mathrm{n}$ & $2-4$ & 2 & Hieracium pilosella & 3.09 & III \\
\hline HRs & $\mathrm{x}$ & 4 & $\mathrm{x}$ & $\mathrm{x}$ & 3 & 4 & 4 & 1 & $\mathrm{n}$ & $2-6$ & 3 & Hypericum perforatum & 0.50 & $\mathrm{I}$ \\
\hline HRs & 6 & 6 & $\mathrm{x}$ & 5 & 4 & 3 & 3 & 4 & $\mathrm{n}$ & $2-3$ & 1 & Inula britanica & 0.50 & I \\
\hline $\mathrm{HR}$ & $\mathrm{x}$ & 5 & $\mathrm{x}$ & 5 & 7 & 7 & 7 & 5 & $\mathrm{n}$ & $3-4$ & 3 & Leontodon autumnalis & 1.17 & I \\
\hline $\mathrm{H}$ & 7 & 3 & 8 & 2 & 4 & 4 & 3 & 4 & $\mathrm{n}$ & $3-4$ & 1 & Linum austriacum & 0.50 & $\mathrm{I}$ \\
\hline TRs & 6 & 5 & 7 & 5 & 6 & 5 & 7 & 3 & $\mathrm{n}$ & $3-5$ & 1 & Odontites lutea & 0.50 & $\mathrm{I}$ \\
\hline HRs & $\mathrm{x}$ & 4 & 8 & 4 & 5 & 4 & 2 & 4 & $\mathrm{n}$ & $2-5$ & 2 & Picris hieracioides & 0.50 & $\mathrm{I}$ \\
\hline HRs & $\mathrm{x}$ & 3 & $\mathrm{x}$ & 2 & 6 & 5 & 5 & 5 & $\mathrm{n}$ & $2-3$ & 1 & Pimpinella saxifraga & 1.25 & II \\
\hline $\mathrm{HR}$ & $\mathrm{x}$ & $\mathrm{x}$ & $\mathrm{x}$ & $\mathrm{x}$ & 7 & 6 & 6 & 6 & $\mathrm{n}$ & $2-4$ & 3 & Plantago lanceolata & 1.83 & IV \\
\hline HR & $\mathrm{x}$ & 4 & 8 & 3 & 4 & 8 & 8 & 5 & $\mathrm{n}$ & $2-4$ & 2 & Plantago media & 3.00 & $\mathrm{~V}$ \\
\hline $\mathrm{H}$ & $\mathrm{x}$ & 2 & 5 & 1 & 3 & 4 & 4 & 4 & $\mathrm{n}$ & $2-4$ & 2 & Potentilla argentea & 2.50 & $\mathrm{I}$ \\
\hline $\mathrm{H}$ & 7 & 1 & 8 & 1 & 3 & 4 & 5 & 4 & $\mathrm{n}$ & $2-3$ & 2 & Potentilla incana & 5.71 & $\mathrm{~V}$ \\
\hline $\mathrm{HS}$ & $\mathrm{x}$ & $\mathrm{x}$ & 4 & $\mathrm{x}$ & 9 & 8 & 8 & 4 & $\mathrm{n}$ & $3-4$ & 2 & Prunella vulgaris & 0.94 & II \\
\hline HRs & - & 8 & 7 & 6 & 3 & 2 & 2 & 4 & $\mathrm{n}$ & $2-3$ & 1 & $\begin{array}{c}\text { Pseudolysimachion } \\
\text { longifolium }\end{array}$ & 0.50 & I \\
\hline $\mathrm{H}$ & 6 & 3 & 7 & 3 & 5 & 3 & 3 & 4 & $\mathrm{n}$ & $3-4$ & 1 & Salvia nemorosa & 1.00 & $\mathrm{I}$ \\
\hline HRs & 6 & 4 & 8 & 4 & 5 & 3 & 3 & 4 & $\mathrm{n}$ & $2-3$ & 2 & Salvia pratensis & 2.64 & IV \\
\hline $\mathrm{H}$ & 6 & 4 & 7 & 5 & 5 & 3 & 3 & 4 & $\mathrm{n}$ & $3-4$ & 2 & Salvia verticillata & 1.17 & II \\
\hline HRs & 6 & 4 & 8 & 3 & 4 & 4 & 5 & 5 & $\mathrm{n}$ & - & - & Sanguisorba minor & 1.00 & $\mathrm{I}$ \\
\hline- & - & - & - & - & - & - & - & - & - & - & - & Scabiosa argentea & 0.50 & II \\
\hline $\mathrm{T}$ & 5 & 5 & 3 & 6 & - & - & - & 4 & $\mathrm{n}$ & $4-5$ & 3 & Spergula arvensis & 0.50 & $\mathrm{I}$ \\
\hline GRs & 5 & 7 & 7 & 6 & 4 & 2 & 2 & 3 & $\mathrm{n}$ & $3-5$ & 2 & Stachis germanica & 0.50 & $\mathrm{I}$ \\
\hline $\mathrm{HR}$ & $\mathrm{x}$ & 5 & $\mathrm{x}$ & 6 & 8 & 7 & 7 & 7 & $\mathrm{n}$ & $3-5$ & 3 & Taraxacum officinale & 0.50 & $\mathrm{I}$ \\
\hline- & - & - & - & - & - & - & - & - & - & - & - & Thymus glabrescens & 3.68 & III \\
\hline $\mathrm{ChL}$ & $\mathrm{x}$ & 4 & 5 & 6 & 4 & 4 & 4 & 3 & $\mathrm{n}$ & $2-3$ & 2 & Thymus pulegioides & 2.64 & II \\
\hline HRs & 5 & 4 & $\mathrm{x}$ & 6 & 3 & 3 & 7 & 4 & $\mathrm{n}$ & $3-4$ & 4 & Verbena officinalis & 0.50 & I \\
\hline $\mathrm{HS}$ & $\mathrm{x}$ & 3 & 5 & 6 & 8 & 7 & 7 & 4 & $\mathrm{n}$ & $3-5$ & 2 & Veronica serpyllifolia & 2.50 & I \\
\hline TT & 7 & 5 & 7 & 6 & 2 & - & 8 & 2 & $\mathrm{n}$ & $5-6$ & - & Xanthium strumarium & 0.50 & $\mathrm{I}$ \\
\hline- & - & - & - & - & - & - & - & - & - & - & - & OBF & 69.57 & \\
\hline
\end{tabular}


Juncaceae families in this type of grassland, we find that they are lacking.

Fabaceae family participates in the floristic composition, an average of only $5.58 \%$, registering a maximum of $1.71 \%$ (Annex 1).

Data collected on Fabaceae participation are symmetric (obliquity $=0.43$ ) and a distribution curvedflat (kurt $=0.49$, Annex 1). From Fabaceae species the Trifolium repens has good participation (1.25\%), followed by species Dorychnium herbaceum (1.17\%) and Medicago lupulina $(1.17 \%)$, other species are with lower covered.

Plants from other botanical families (OBF) are present in Festuca rupicola -Bothriochloa ischaemum phytocoenosis type on average by $69.57 \%$, reaching a minimum of $0.61 \%$ and a maximum of $3.02 \%$ (Annex 1). Data obtained on the participation of plants from other botanical families are symmetrical, positive (obliquity = 0.43 ) and platicurtic arching $=-0.91$, Annex 1) .

Some species in this group have a significant mean abundance such as: Fragaria vesca $(7.53 \%)$, Potentilla incana with $5.71 \%$ coverage, Adonis vernalis (5.25\%), Thymus glabrescens with $3.68 \%$,
Hieracium pilosella with $3.09 \%$, and Plantago media with $3.00 \%$ (Tab. 1).

Some species have a lower participation on average of 2\%, as species: Euphorbia cyparissias, Potentilla argentea, Salvia pratensis, Thymus pulegioides and Veronica serpyllifolia. Many species have a low participation on average $0.5-1 \%$.

From an environmental (ecological spectrum) phytocoenosis is of a meso-xerofil ( $\mathrm{Up}=3.5)$, neutrophil $(\mathrm{Rp}=7.2)$ and have a character oligomezotrophic ( $\mathrm{Np}=3.5$; Tab. 2).

From agronomical point of view (agronomic spectrum), the phytocoenosis is medium tolerant of mowing ( $\mathrm{Cp}=5.0)$, is medium grazing tolerant $(\mathrm{Pp}=5.6)$ and medium crushed tolerant $(\mathrm{Sp}=5.3$, Tab. 2).

Thus, the fodder value of Festuca rupicola -Bothriochloa ischaemum type is 3.9 (VFP $=3.9$, Tab. 2), so the grassland falling within Class III, category of degraded pasture and supports 0.21 to $0.40 \mathrm{LU} / \mathrm{ha}$.

From agronomical point of view, in studied phytocoenosis, 5 species have toxic value with a total coverage of $9.7 \%$ (Dorychnium herbaceum,

Tab. 2. Ecological and agronomical spectrum of Festuca rupicola -Bothriochloa ischaemum grassland type

\begin{tabular}{|c|c|c|c|c|c|c|c|c|c|c|c|}
\hline \multirow{2}{*}{ Eclg. indexes } & \multicolumn{10}{|c|}{ Ecological spectrum } & \multirow{2}{*}{$\begin{array}{l}\text { VIMnp } \\
\text { VIMp }\end{array}$} \\
\hline & 1 & 2 & 3 & 4 & 5 & 6 & 7 & 8 & 9 & $\mathbf{x}$ & \\
\hline Unp & 1.0 & 5.0 & 12.0 & 29.0 & 12.0 & 4.0 & 1.0 & 1.0 & 0.0 & 14 & 4.0 \\
\hline Up & 5.7 & 10.5 & 50.3 & 36.4 & 15.9 & 3.7 & 0.5 & 0.5 & 0.0 & 19.87 & 3.5 \\
\hline Rnp & 0.0 & 3.0 & 1.0 & 2.0 & 5.0 & 3.0 & 18.0 & 16.0 & 0.0 & 31.00 & 6.5 \\
\hline $\mathbf{R p}$ & 0.0 & 1.5 & 0.5 & 1.7 & 8.9 & 1.5 & 18.6 & 51.7 & 0.0 & 59.01 & 7.2 \\
\hline Nnp & 4.0 & 9.0 & 11.0 & 9.0 & 8.0 & 24.0 & 2.0 & 1.0 & 0.0 & 11.00 & 4.4 \\
\hline $\mathrm{Np}$ & 14.0 & 35.4 & 32.2 & 12.1 & 6.9 & 28.5 & 2.7 & 0.7 & 0.0 & 11.01 & 3.5 \\
\hline \multirow{2}{*}{$\begin{array}{l}\text { Agron. } \\
\text { indexes }\end{array}$} & \multicolumn{10}{|c|}{ Agronomic spectrum } & VIMnp \\
\hline & 1 & 2 & 3 & 4 & 5 & 6 & 7 & 8 & 9 & $\mathrm{x}$ & VIMp \\
\hline Cnp & 0.0 & 4.0 & 10.0 & 20.0 & 12.0 & 9.0 & 9.0 & 6.0 & 2.0 & 0.00 & 4.9 \\
\hline $\mathrm{Cp}$ & 1.0 & 8.4 & 25.3 & 21.1 & 25.2 & 5.7 & 30.5 & 8.3 & 1.4 & 0.00 & 5.0 \\
\hline Pnp & 0.0 & 9.0 & 9.0 & 21.0 & 6.0 & 5.0 & 12.0 & 8.0 & 2.0 & 0.00 & 4.9 \\
\hline Pp & 0.0 & 5.5 & 15.3 & 32.4 & 4.7 & 9.1 & 34.8 & 23.2 & 1.0 & 0.00 & 5.6 \\
\hline Snp & 0.0 & 11.0 & 12.0 & 15.0 & 7.0 & 7.0 & 13.0 & 7.0 & 2.0 & 0.00 & 4.9 \\
\hline Sp & 0.0 & 6.5 & 26.7 & 19.9 & 12.2 & 10.8 & 37.9 & 11.9 & 1.0 & 0.00 & 5.3 \\
\hline FVnp & 5.0 & 4.0 & 6.0 & 25.0 & 10.0 & 4.0 & 3.0 & 3.0 & 1.0 & 0 & 4.2 \\
\hline FVp & 9.7 & 4.9 & 24.49 & 60.4 & 13.7 & 5.0 & 1.5 & 2.3 & 2.8 & 0 & 3.9 \\
\hline
\end{tabular}

Legend

U humidity

$\begin{array}{ll}\mathbf{R} & \text { soil reaction } \\ \mathbf{N} & \text { nutrition }\end{array}$

$\begin{array}{ll}\mathbf{C} & \text { mowing } \\ \mathbf{P} & \text { grazing }\end{array}$
crushed
VF fodder value

np unweighted (depending on the number of species)

p weighted (depending on species coverage) 
Adonis vernalis, Colchium autumnale, Euphorbia cyparissias and Hypericum perforatum; Tab. 1) and 4 species are harmful of animal products: Carduus acanthoides (0.70\%), Dipsacus fullonum (1.50\%), Eryngium campestre (1.72\%) and Xanthium strumarium (0.50\%, Tab. 1).

Phytocoenosis is composed of 6 species damaging the grassland vegetation: Bothriochloa ischaemum (13.79\%), Stipa capillata (5.71\%), Agrimonia eupatoria (1.33\%), Odontites lutea (0.52\%), Stachis germanica $(0.50 \%)$ and Thymus pulegioides (2.64\%, Tab. 1).

Phytocoenosis shows high coverage with poor forage species (ballast) with a share of $60.4 \%$. The species of medium fooder have a participation of $18.7 \%$ and the best feed species are in number of 4 and have a cumulative $5.10 \%$ coverage (Tab. 2). There are no species of excellent fodder.

\section{CONCLUSION}

Festuca rupicola-Bothriochloa ischaemum appears on downhill, dry land with a neutral reaction and poor trophicity.

Maintenance work is hardly and seldom implemented, the proof being the presence of molehills and woody vegetation.

Agronomic value of the pasture of Festuca rupicola- Bothriochloa ischaemum is mediocre, being predominant by ballast species with reduced grazing capacity.

Grasslands are used in a semi-extensive system, plants being trampled frequently in grazing period.

We recommend a management pastoral developing plan which should contain adequate maintenance and a system of sustainable use.

\section{REFERENCES}

1. Cristea V, Gafta D, Pedrotti F (2004). Fitosociology. Publisher Presa Universitară Clujeană (Chapter IV).

2. Fenton JHC (2008). A postulated natural origin for the landscape of upland Scotland. Plant Ecology \& Diversity 1:115-127.

3. Gordon IJ, Hester AJ, Festa-Bianchet M (2004). The management of wild large herbivores to meet economic, conservation and environmental objectives. Journal of Applied Ecology 41:1021-1031.

4. Herbei M (2011). Elemente metodologice aplicate in cercetarea pajistilor. Printhouse Mirton Timisoara.

5. Pollock ML, Holland JP, Morgan-Davies C, Morgan-Davies J and Waterhouse A (2013). Reduced Sheep Grazing and Biodiversity: A Novel Approach to Selecting and Measuring Biodiversity. Rangeland Ecol. Manage 66:387-400. July 2013. DOI: 10.2111/REM-D-11-00123.1 Indicators.

6. Miles J (1988). Vegetation and soil change in the uplands. In: M. B. Usher and D. B. A. Thompson [EDS.]. Ecological change in the uplands. Oxford, UK: Blackwell Scientific Publications. British Ecological Society Special Publication 7:57-70.

7. Păcurar F, Rotar I (2014). Metode de studiu și interpretare a vegetației pajiștilor. Printhouse Risoprint, Cluj-Napoca, ISBN 978-973-53-1452-1.

8. Păcurar F, Rotar I, Balaszi A, Pleșa A, Gliga A, Vaida I, Guita A (2016). The pastoral management plan of Frata commune, unpublished matherial.

9. Pușcaru-Soroceanu Evdochia, Pușcaru D, Buia A, Burduja C, Csuros S,, Grâneanu A, Niedermayer K, Popescu P, Răvăruț M, Resmeriță I, Samiolă Z, Vasiu V, Velea C (1963). Grassland and haymeadows from R.P.R. - Geobotany and agroproductive study. Printhouse of R.P.R Academy.

10. Rotar I, Păcurar F, Balaszi A, Pleșa A, Gliga A, Vaida I, Guita A (2016). The pastoral management plan of Aiton commune, unpuplished matherial.

11. Țucra I, Kovacs AJ, Roșu C, Ciubotaru C, Chifu T, Neacșu M, Bărbulescu C, Cardașol V, Popovici D, Simtea N, Motcă G, Dragu I, Spirescu M (1987),-- Principalele tipuri de pajiști din R.S. România, Centrul de Material Didactic și Propagandă Agricolă, București.

12. (http://statisticasociala.tripod.com/parametri.htm). 
Annex 1. Statistic processing of data regarding stand conditions, species composition of Festuca rupicola -Botriochloa ischaemum type and the species' frequence constancy in relevees

\begin{tabular}{|c|c|c|c|c|c|c|c|c|c|c|}
\hline Species & $\overline{\mathbf{x}}$ & Median & Stdev & VC & Mode & Skew & Kurt & Min & Max & Count \\
\hline Agrostis capillaris & 0.90 & 0.50 & 0.89 & 0.99 & 0.50 & 2.24 & 5.00 & 0.50 & 2.50 & 5 \\
\hline $\begin{array}{l}\text { Bothriochloa } \\
\text { ischaemum }\end{array}$ & 13.79 & 12.50 & 3.79 & 0.27 & 12.50 & 0.67 & 2.03 & 5.00 & 22.25 & 22 \\
\hline Brachipodium pinnatum & 3.50 & 2.50 & 1.37 & 0.39 & 2.50 & 0.61 & -3.33 & 2.50 & 5.00 & 5 \\
\hline Cynodon dactylon & 0.50 & 0.50 & 0.00 & 0.00 & 0.00 & 0.00 & 0.00 & 0.50 & 0.50 & 1 \\
\hline Cynosurus cristatus & 0.50 & 0.50 & 0.00 & 0.00 & 0.50 & 0.00 & 0.00 & 0.50 & 0.50 & 2 \\
\hline Elymus elongatus & 3.10 & 2.50 & 1.92 & 0.62 & 5.00 & -0.25 & -1.33 & 0.50 & 5.00 & 5 \\
\hline Festuca rupicola & 21.81 & 22.25 & 4.88 & 0.22 & 22.25 & 0.23 & -0.74 & 12.50 & 29.00 & 22 \\
\hline Festuca valesiaca & 1.25 & 0.50 & 1.04 & 0.83 & 0.50 & 0.64 & -2.24 & 0.50 & 2.50 & 8 \\
\hline Phleum pratense & 2.75 & 2.75 & 3.18 & 1.16 & 0.00 & 0.00 & 0.00 & 0.50 & 5.00 & 2 \\
\hline Poa pratensis & 0.50 & 0.50 & 0.00 & 0.00 & 0.00 & 0.00 & 0.00 & 0.50 & 0.50 & 1 \\
\hline Stipa capillata & 5.71 & 8.00 & 3.13 & 0.55 & 8.00 & -0.97 & -0.78 & 0.50 & 8.00 & 8 \\
\hline POACEAE & 4.94 & 1.84 & & & 4.70 & 0.29 & -0.13 & 2.18 & 7.34 & 7.36 \\
\hline $\begin{array}{l}\text { CYPERACEAE- } \\
\text { JUNCACEAE }\end{array}$ & 0.00 & 0.00 & 0.00 & 0.00 & 0.00 & 0.00 & 0.00 & 0.00 & 0.00 & 0 \\
\hline $\begin{array}{c}\text { Astragalus } \\
\text { monspessulanus }\end{array}$ & 0.50 & 0.50 & 0.00 & 0.00 & 0.50 & 0.00 & 0.00 & 0.50 & 0.50 & 5 \\
\hline Dorychnium herbaceum & 1.17 & 0.50 & 1.15 & 0.99 & 0.50 & 1.73 & 0.00 & 0.50 & 2.50 & 3 \\
\hline Lotus corniculatus & 0.50 & 0.50 & 0.00 & 0.00 & 0.50 & 0.00 & 0.00 & 0.50 & 0.50 & 5 \\
\hline Medicago lupulina & 1.17 & 0.50 & 1.15 & 0.99 & 0.50 & 1.73 & 0.00 & 0.50 & 2.50 & 3 \\
\hline Trifolium medium & 0.50 & 0.50 & 0.00 & 0.00 & 0.50 & 0.00 & 0.00 & 0.50 & 0.50 & 3 \\
\hline Trifolium pratense & 0.50 & 0.50 & 0.00 & 0.00 & 0.50 & 0.00 & 0.00 & 0.50 & 0.50 & 3 \\
\hline Trifolium repens & 1.25 & 0.50 & 1.37 & 1.10 & 0.50 & 1.90 & 3.41 & 0.50 & 5.00 & 15 \\
\hline FABACEAE & 0.80 & 0.53 & 0.44 & 0.50 & 0.50 & 0.77 & 0.49 & 0.50 & 1.71 & 5.29 \\
\hline Achillea millefolium & 1.81 & 1.50 & 1.84 & 1.02 & 0.50 & 2.34 & 7.35 & 0.50 & 8.00 & 19 \\
\hline Adonis vernalis & 5.25 & 5.25 & 3.89 & 0.74 & 0.00 & 0.00 & 0.00 & 2.50 & 8.00 & 2 \\
\hline Agrimonia eupatoria & 1.33 & 0.50 & 1.03 & 0.77 & 0.50 & 0.39 & -2.26 & 0.50 & 2.50 & 13 \\
\hline Campanula serpilifolia & 0.50 & 0.50 & 0.00 & 0.00 & 0.00 & 0.00 & 0.00 & 0.50 & 0.50 & 1 \\
\hline Carduus acanthoides & 0.70 & 0.50 & 0.63 & 0.90 & 0.50 & 3.16 & 10.00 & 0.50 & 2.50 & 10 \\
\hline Centaurea jacea & 1.50 & 1.50 & 1.41 & 0.94 & 0.00 & 0.00 & 0.00 & 0.50 & 2.50 & 2 \\
\hline Centaurea nigrescens & 0.50 & 0.50 & 0.00 & 0.00 & 0.00 & 0.00 & 0.00 & 0.50 & 0.50 & 2 \\
\hline Clinopodium vulgare & 0.50 & 0.50 & 0.00 & 0.00 & 0.50 & 0.00 & 0.00 & 0.50 & 0.50 & 2 \\
\hline Colchium autumnale & 0.50 & 0.50 & 0.00 & 0.00 & 0.00 & 0.00 & 0.00 & 0.50 & 0.50 & 1 \\
\hline Cichorium intybus & 0.79 & 0.50 & 0.76 & 0.96 & 0.50 & 2.65 & 7.00 & 0.50 & 2.50 & 7 \\
\hline Crepis biennis & 0.50 & 0.50 & 0.00 & 0.00 & 0.00 & 0.00 & 0.00 & 0.50 & 0.50 & 1 \\
\hline Daucus carota & 0.50 & 0.50 & 0.00 & 0.00 & 0.50 & 0.00 & 0.00 & 0.50 & 0.50 & 3 \\
\hline Dipsacus fullonum & 1.50 & 1.50 & 1.41 & 0.94 & 0.00 & 0.00 & 0.00 & 0.50 & 2.50 & 2 \\
\hline Erodium hoefftianum & 0.00 & 0.00 & 0.00 & 0.00 & 0.00 & 0.00 & 0.00 & 0.00 & 0.00 & 0 \\
\hline Eryngium campestre & 1.72 & 2.50 & 1.00 & 0.58 & 2.50 & -0.50 & -1.99 & 0.50 & 2.50 & 19 \\
\hline Euphorbia cyparissias & 2.32 & 2.50 & 1.32 & 0.57 & 2.50 & 0.43 & 0.72 & 0.50 & 5.00 & 18 \\
\hline Filipendula hexapetala & 1.50 & 1.50 & 1.10 & 0.73 & 2.50 & 0.00 & -3.33 & 0.50 & 2.50 & 7 \\
\hline Fragaria vesca & 7.53 & 6.50 & 5.16 & 0.69 & 5.00 & 0.56 & -0.55 & 0.50 & 17.50 & 21 \\
\hline Galium verum & 0.50 & 0.50 & 0.00 & 0.00 & 0.50 & 0.00 & 0.00 & 0.50 & 0.50 & 2 \\
\hline Hieracium pilosella & 3.09 & 2.50 & 2.17 & 0.70 & 2.50 & 1.16 & 1.65 & 0.50 & 8.00 & 11 \\
\hline
\end{tabular}




\begin{tabular}{|c|c|c|c|c|c|c|c|c|c|c|}
\hline Species & $\overline{\mathbf{x}}$ & Median & Stdev & VC & Mode & Skew & Kurt & Min & Max & Count \\
\hline Hypericum perforatum & 0.50 & 0.50 & 0.00 & 0.00 & 0.00 & 0.00 & 0.00 & 0.50 & 0.50 & 1 \\
\hline Inula britanica & 0.50 & 0.50 & 0.00 & 0.00 & 0.50 & 0.00 & 0.00 & 0.50 & 0.50 & 2 \\
\hline Leontodon autumnalis & 1.17 & 0.50 & 1.15 & 0.99 & 0.50 & 1.73 & 0.00 & 0.50 & 2.50 & 3 \\
\hline Linum austriacum & 0.50 & 0.50 & 0.00 & 0.00 & 0.00 & 0.00 & 0.00 & 0.50 & 0.50 & 1 \\
\hline Odontites lutea & 0.50 & 0.50 & 0.00 & 0.00 & 0.00 & 0.00 & 0.00 & 0.50 & 0.50 & 1 \\
\hline Picris hieracioides & 0.50 & 0.50 & 0.00 & 0.00 & 0.50 & 0.00 & 0.00 & 0.50 & 0.50 & 2 \\
\hline Pimpinella saxifraga & 1.25 & 0.50 & 1.04 & 0.83 & 0.50 & 0.64 & -2.24 & 0.50 & 2.50 & 8 \\
\hline Plantago lanceolata & 1.83 & 2.50 & 0.98 & 0.53 & 2.50 & -0.79 & -1.62 & 0.50 & 2.50 & 16 \\
\hline Plantago media & 3.00 & 2.50 & 2.44 & 0.81 & 2.50 & 0.95 & 0.20 & 0.50 & 8.00 & 18 \\
\hline Potentilla argentea & 2.50 & 2.50 & 0.00 & 0.00 & 0.00 & 0.00 & 0.00 & 2.50 & 2.50 & 1 \\
\hline Potentilla incana & 5.71 & 5.00 & 3.96 & 0.69 & 2.50 & 0.52 & -0.85 & 0.50 & 12.50 & 19 \\
\hline Prunella vulgaris & 0.94 & 0.50 & 0.98 & 1.05 & 0.50 & 1.29 & -0.11 & 0.00 & 2.50 & 8 \\
\hline $\begin{array}{c}\text { Pseudolysimachion } \\
\text { longifolium }\end{array}$ & 0.50 & 0.50 & 0.00 & 0.00 & 0.00 & 0.00 & 0.00 & 0.50 & 0.50 & 1 \\
\hline Salvia nemorosa & 1.00 & 0.50 & 1.00 & 1.00 & 0.50 & 0.52 & 4.00 & 0.50 & 2.50 & 4 \\
\hline Salvia pratensis & 2.64 & 2.50 & 1.77 & 0.67 & 2.50 & 0.21 & -1.25 & 0.50 & 5.00 & 14 \\
\hline Salvia verticillata & 1.17 & 0.50 & 1.03 & 0.89 & 0.50 & 0.97 & -1.88 & 0.50 & 2.50 & 7 \\
\hline Sanguisorba minor & 1.00 & 0.50 & 1.00 & 1.00 & 0.50 & 2.00 & 4.00 & 0.50 & 2.50 & 4 \\
\hline Scabiosa argentea & 0.50 & 0.50 & 0.00 & 0.00 & 0.50 & 0.00 & 0.00 & 0.50 & 0.50 & 9 \\
\hline Spergula arvensis & 0.50 & 0.50 & 0.00 & 0.00 & 0.50 & 0.00 & 0.00 & 0.50 & 0.50 & 3 \\
\hline Stachis germanica & 0.50 & 0.50 & 0.00 & 0.00 & 0.50 & 0.00 & 0.00 & 0.50 & 0.50 & 2 \\
\hline Taraxacum officinale & 0.50 & 0.50 & 0.00 & 0.00 & 0.00 & 0.00 & 0.00 & 0.50 & 0.50 & 1 \\
\hline Thymus glabrescens & 3.68 & 2.50 & 4.01 & 1.09 & 2.50 & 1.34 & 0.89 & 0.50 & 12.50 & 11 \\
\hline Thymus pulegioides & 2.64 & 2.50 & 1.84 & 0.70 & 2.50 & 0.24 & -1.19 & 0.50 & 5.00 & 8 \\
\hline Verbena officinalis & 0.50 & 0.50 & 0.00 & 0.00 & 0.00 & 0.00 & 0.00 & 0.50 & 0.50 & 1 \\
\hline Veronica serpyllifolia & 2.50 & 2.50 & 0.00 & 0.00 & 2.50 & 0.00 & 0.00 & 2.50 & 2.50 & 2 \\
\hline Xanthium strumarium & 0.50 & 0.50 & 0.00 & 0.00 & 0.50 & 0.00 & 0.00 & 0.50 & 0.50 & 2 \\
\hline OBF & 1.51 & 0.93 & 0.43 & 1.34 & 0.91 & 0.43 & 0.40 & 0.61 & 3.02 & 6.35 \\
\hline
\end{tabular}

Legend: $\overline{\mathbf{x}}$-Average; St.dev. - Standard deviation; VC - variability coefficient; OBF-other botanical families 